\title{
Control of the Marangoni Effect in the Drying Process of a Polymer Solution Coated on a Flat Substrate through Temperature, Evaporation and Solute Concentration Management
}

\author{
By Hiroyuki Kagami*
}

\begin{abstract}
The drying process of polymer solutions coated on a flat substrate is very important in various industrial applications. We have proposed and modified a model of the drying process of a polymer solution coated on a flat substrate for uniform polymer film deposition. We used the model to control the thickness of a thin film after drying through management of the temperature, evaporation, and concentration. We incorporated the Marangoni effect into the existing model in the form of pseudo-negative diffusion at an upper gas-liquid interface. We applied the thermal, evaporative, and concentration management to the modified model incorporating the Marangoni effect. The results demonstrated that our method of thickness control of a thin film after drying was effective for improving the Marangoni effect.
\end{abstract}

\section{Introduction}

The drying of polymer solutions coated on a flat substrate is very important in various industrial applications such as fabricating flat polymer thin films (Bornside et al., 1989; Routh and Russel, 1998) and inkjet printing (de Gans and Schubert, 2004; Soltman and Subramanian, 2008). There are various studies of this process. Previous studies of the fabrication of flat polymer thin films have focused on the temporal variation in the thickness of polymer liquid films during drying (Bornside et al., 1989; Routh and Russel, 1998), without considering spatiotemporal variation in concentration distribution. Previous studies of inkjet printing have discussed droplets of polymer solution (de Gans and Schubert, 2004; Soltman and Subramanian, 2008), and thus have a different scope from our study, which considers a largearea liquid film of polymer solution.

We have studied the drying of a large-area liquid film of a polymer solution on a flat substrate by examining the spatiotemporal variation in concentration distribution during drying (Kagami, 2003; Kagami, 2004a; Kagami, 2004b; Kagami, 2007; Kagami, 2008; Kagami, 2009; Kagami, 2010; Kagami, 2014; Kagami and Kubota, 2010; Kagami et al., 2002). We showed

*Associate Professor, Fujita Health University, Japan. 
that the distribution of polymer molecules on the substrate after drying is described by a power relation between the vaporization rate, the diffusion coefficient of the solvent containing the solutes, and the diffusion coefficient of the concentration of the solution.

We have proposed and modified a model of the drying process of a polymer solution coated on a flat substrate for flat polymer film fabrication (Kagami et al., 2002; Kagami, 2003; Kagami, 2004a; Kagami, 2004b; Kagami, 2007; Kagami, 2008; Kagami, 2009; Kagami, 2010; Kagami and Kubota, 2010a). In addition, we clarified the dependence of the distribution of polymer molecules on a flat substrate after drying on a various parameters based on the analysis of many numerical simulations of the model.

However, the mechanism of the drying process in the previous studies does not allow problems with thin films after drying, such as thickening of the edges and depressions near the edge, to be avoided. We applied the model to thickness control of a thin film after drying through thermal, evaporative, and solute concentration management (Kagami, 2014; Kagami and Kubota, 2010b; Kagami and Kubota, 2011). A film thickness of approximately $300 \mathrm{~nm}$ was assumed, which is similar to films for resist coating of wafers and reticle substrates in semiconductor engineering. During these processes, thickening at the edge and depressions next to the edge of the film should be reduced to several percent or less to make the films suitable for a wide range of applications. Our results showed that control of the thickness of a thin film after drying can be achieved by adding evaporative, thermal and solute concentration operations that depend on the solute concentration distribution on the substrate during drying (Kagami, 2014a).

However, the Marangoni effect occurs during drying. Thus, we incorporated the Marangoni effect into the existing model in the form of pseudo-negative diffusion at an upper gas-liquid interface. As a result, we demonstrated that the solutes that exist at the upper gas-liquid interface are attracted more strongly to the edge due to the Marangoni effect (Kagami, 2014b).

In this study, we apply the thermal, evaporative, and solute concentration management to the modified model that includes the Marangoni effect. We found that the thickening of the edge of the thin film after drying caused by the Marangoni effect can be reduced by using our method.

\section{Model}

\section{Drying Process}

A series of studies has been conducted on the drying process of polymer (resist) solutions coated on flat substrates under reduced pressure for flat and homogeneous resist film fabrication using photolithography (Kagami, 2011; Kagami, 2014a; Kagami, 2014b). After the resist solution is applied as a flat coating on a flat substrate by scan coating, the coated resist solution is dried under reduced pressure or vacuum. The latter process is the subject of this 
study. However, it is not limited to resist solutions, and we consider general polymer solutions in this study.

Because of the reduced pressure, we can ignore the effect of gas flow over the flat substrate. For sufficiently low gas flow under reduced pressure, disturbances to the interface between the liquid film on the flat substrate and the gas are effectively eliminated. In addition, if the layer of solution film on the flat substrate is extremely thin, for example, if its Rayleigh number is smaller than the critical Rayleigh number, we can also ignore the influence of the advective term of the Navier-Stokes equation. Therefore, the drying process can be described by the following simple model.

\section{Theory and Basic Equations}

Because details of theory and the basic equations have been reported in previous papers (Kagami, 2011; Kagami, 2014a; Kagami and Kubota, 2011; Kagami et al., 2002), only the basic equations are shown here.

First, we consider the following evaporation model (Kagami et al., 2002)

$$
G=\gamma(1-\beta C)
$$

Here, $G$ is the evaporation rate, $C$ is the concentration of the solution, and $\beta$ is a constant. Furthermore,

$$
\gamma=K \sqrt{\frac{M}{2 \pi R T}} P_{0}
$$

is a correction factor, where $P_{0}$ is the vapor pressure, $M$ is the molecular weight, $R$ is the gas constant, $T$ is temperature, and $K$ is a correction factor for the theoretical evaporation rate (Hickman and Trevoy, 1952).

Then, the two diffusion models are formulated as follows. First, the diffusion equation for solvent containing solutes is written as

$$
\frac{\partial V}{\partial t}=K_{v} \nabla^{2} V
$$

where $V$ is the volume of solvent containing solutes included in a space and $K_{v}$ is the diffusion coefficient of the solvent (Kagami, 2011). An evaporation term (Eq. (1)) is added to Eq. (3), which describes the interface between liquid and gas (vacuum), so Eq. (3) is modified to (Kagami, 2011)

$\frac{\partial V}{\partial t}=K_{v} \nabla^{2} V-\gamma(1-\beta C)$

Next, the diffusion equation governing the change in concentration in solution is written as (Kagami, 2014a; Kagami and Kubota, 2011)

$$
\frac{\partial N}{\partial t}=K_{C} \nabla^{2} N+\frac{N}{V}\left(K_{V}-K_{C}\right) \nabla^{2} V-\frac{2 K_{C}}{V} \nabla N \cdot \nabla V
$$

where $N$ denotes the number of solute molecules included in a space and $K_{C}$ is the diffusion coefficient of the solution.

In this study, we consider a solution containing one type of solute and one type of solvent for simplicity. Therefore, we build our model mainly using Eqs. (1) - (5).

The diffusion coefficient, $K_{C}$, of the solution changes with time. As mentioned in previous studies, $K_{C}$ is written as (Kagami, 2011) 
Vol. 2, No. $1 \quad$ Kagami: Control of the Marangoni Effect in the Drying Process...

$$
K_{C}=\frac{k_{B} T}{6 \pi R \eta_{0}\{[\eta] C+1\}}
$$

where $k_{B}$ is the Boltzmann constant, $\eta_{0}$ is the viscosity of the solvent, and $[\eta]$ is the intrinsic viscosity.

\section{The Marangoni Effect}

We incorporate the Marangoni effect into the existing model and treat the effect as pseudo-negative diffusion at an upper gas-liquid interface (Kagami, 2014b). Specifically, pseudo-negative diffusion is taken as $\lambda K_{C}$, where, for example, $\lambda=-1.0 \times 10^{-3}$.

\section{Objective and Method}

In previous studies, the dependence of the distribution of polymer molecules on a flat substrate after drying on various parameters, such as the vaporization rate and solute concentration coefficient, was clarified based on the analysis of many numerical simulations of the model. We found that the distribution of polymer molecules on the substrate after drying was determined by a power relation between the vaporization rate, the diffusion coefficient of the solvent containing solutes, and the concentration diffusion coefficient of the solution. Here, we control the vaporization rate and the concentration diffusion coefficient of the solution by managing the temperature, evaporation, and solute concentration of a solution film on the substrate.

If the integrated number of solute polymer molecules at a point on the substrate is more than (less than) $\alpha_{k}$ times the initial average during drying, the temperature of the surface of the polymer solution film over the point is perturbed upward (downward) by $\beta_{k}$. In addition to raising or lowering the point temperature, if the integrated number of solute polymer molecules at a point on the substrate exceeds $\alpha_{k}$ times the initial average during drying, the correction constant $\gamma$ in Eq. (2) over the point is perturbed downward by $\gamma_{k}$. Furthermore, if the integrated number of solute polymer molecules at a point on the substrate exceeds $\alpha_{k}$ times the initial average during drying, the solute concentration of the surface of the polymer solution film over the point is perturbed downward by $\xi_{k}^{-1}$. For example, supposing $\alpha_{1}=1.1, \beta_{1}=1.00005$, and $\gamma_{1}=0.9999$, if the integrated number of polymer molecules at a point on the substrate exceeds 1.1 times the initial average during drying, then the temperature of the surface of the polymer solution film over the point is perturbed upward by 1.00005 and the correction constant $\gamma$ in Eq. (2) over the point is perturbed downward by 0.9999 . 


\section{Results and Discussion}

\section{Numerical Simulation Conditions}

A two-dimensional liquid film of a polymer solution on a flat substrate is modeled. The thickness of the polymer solution is $0.1 \mathrm{~mm}$ and its width is 150 $\mathrm{mm}$. Solvent vaporizes only at the interface between liquid and gas (vacuum) and there is no boiling. The volume of the solvent, $V$, and the number of solute molecules, $N$, in solution are homogeneous, except for the surface in contact with the gas (vacuum). A homogeneous random number is used to produce a $10 \%$ variation in $N$ in the upper surface of the liquid film of the polymer solution.

The simulation is ended when the total volume of the solvent is less than a fixed value, the solute cannot move in solution, and drying is complete. The fixed value is determined indirectly from experimental data.

The initial values of parameters are set as $K_{C}=1.8750 \times 10^{-8} \mathrm{~m}^{2} / \mathrm{s}$, $K_{v}=5.9375 \times 10^{-8} \mathrm{~m}^{2} / \mathrm{s}, \gamma=5.7000 \times 10^{-14} \mathrm{~m}^{2} / \mathrm{s}$.

In this trial, we set the parameters $\alpha_{k}, \beta_{k}, \gamma_{k}$ and $\xi_{k}$ as shown in Table 1.

Table 1. Parameter Settings in the Numerical Simulation

\begin{tabular}{|c|c|}
\hline$\alpha_{1}$ & (over) 1.1 \\
\hline$\alpha_{2}$ & (over) 1.02 \\
\hline$\alpha_{3}$ & (under) 0.98 \\
\hline$\alpha_{4}$ & (under) 0.96 \\
\hline$\beta_{1}$ & 1.00005 \\
\hline$\beta_{2}$ & 1.000025 \\
\hline$\beta_{3}$ & 0.999997 \\
\hline$\beta_{4}$ & 0.99999 \\
\hline$\gamma_{1}$ & 0.9999 \\
\hline$\gamma_{2}$ & 0.99999 \\
\hline$\xi_{1}$ & 1.001 \\
\hline$\xi_{2}$ & 1.0005 \\
\hline
\end{tabular}

Numerical Simulation Results

A typical thickness profile of a polymer thin film after drying is shown in Figure 1. 
Vol. 2, No. $1 \quad$ Kagami: Control of the Marangoni Effect in the Drying Process...

Figure 1. Typical Thickness Profile of a Resist Film after Drying (Kagami et al., 2002)

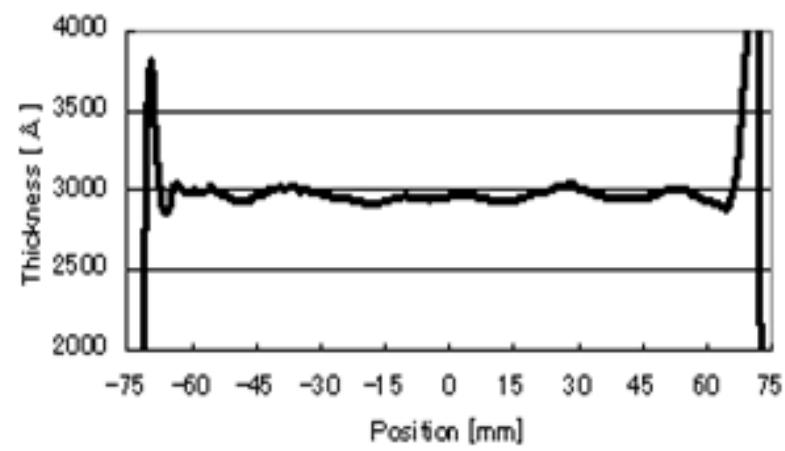

Figure 1. These are experimental data for a resist film. The horizontal axis shows the position from the center of the substrate surface and the vertical axis shows the thickness of the resist film. The edge of the thin film is much thicker than the middle, and the region next to the edge is slightly thinner. This unevenness at the edge can be improved by using our method.

In Figure 2, we show the solute distribution after drying by using our method.

Figure 2. Improvement of Solute Distribution after Drying by using our Method

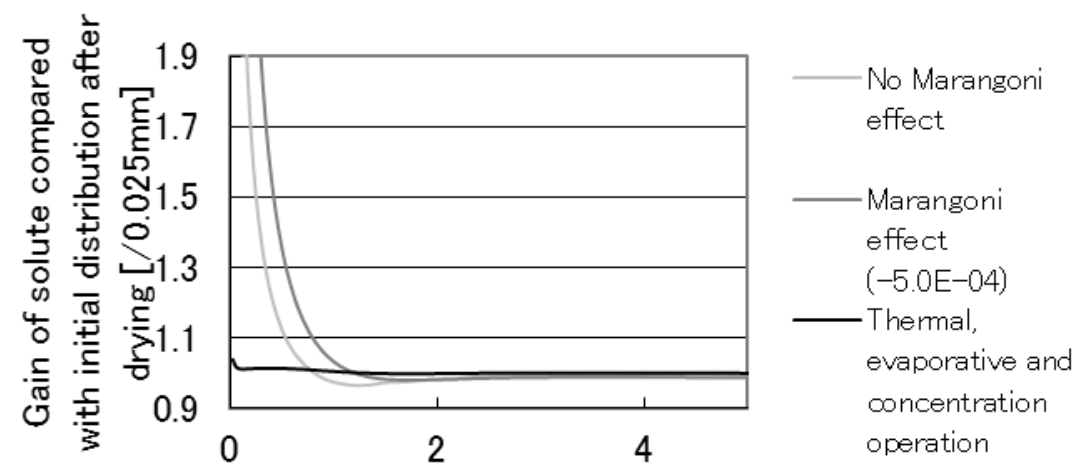

Position from the edge of thin film after drying [mm]

The thickening at the edge is greatly improved although Figure 3 shows that the improvement is greater when the Marangoni effect is included. This can be explained as follows. 
Figure 3. Improvement of Solute Distribution after Drying by our Method

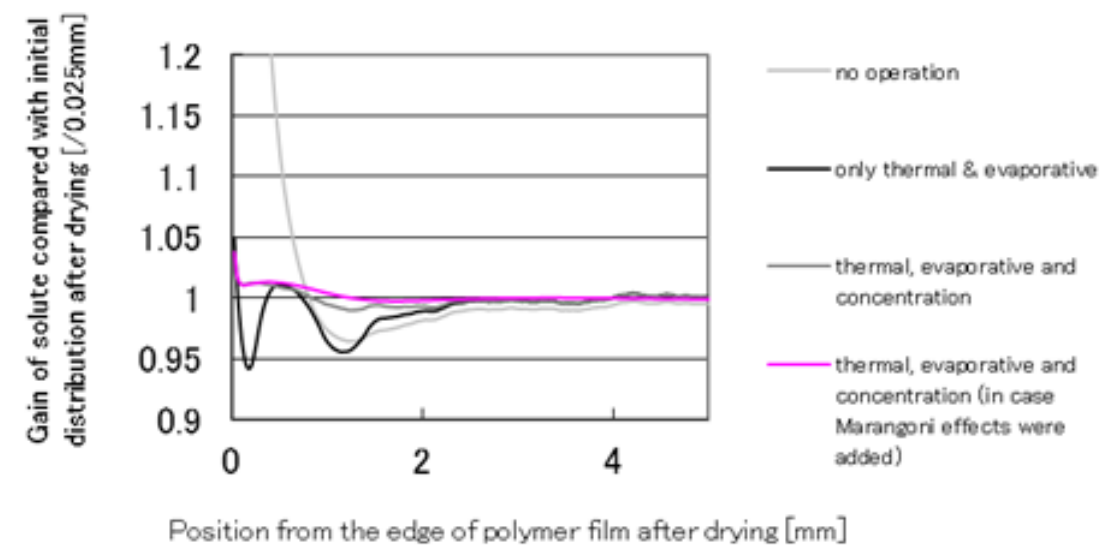

If the integrated number of polymer molecules at a point on the substrate increases, the temperature of the polymer solution film surface over the point should be perturbed upward. Then the diffusion coefficient of the solution becomes larger. As a result, the integrated number of polymer molecules at the point decreases because the solutes diffuse outward due to the larger diffusion coefficient of the solution. Conversely, if the integrated number of polymer molecules at a point on the substrate decreases, the temperature should be decreased. Moreover, if the integrated number of polymer molecules at a point on the substrate increases, the evaporation rate at the point should be decreased slightly so as to avoid the solute's gathering there due to the diffusion of solvent containing solutes. Lastly, if the integrated number of polymer molecules at a point on the substrate increases, the solute concentration at the point should be decreased slightly so as to prompt the solutes at the point to diffuse outward.

\section{Conclusions}

In this study, we applied thermal, evaporative and solute concentration management to a modified model that includes the Marangoni effect. The thickening at the edge of the thin film after drying due to the Marangoni effect can be improved through appropriate thermal, evaporative and solute concentration management. Our method of thickness control of a thin film after drying is effective for countering the Marangoni effect. Control of the film thickness distribution after drying by precisely managing temperature, evaporation, and solute concentration needs to be experimentally validated in future work. 
Vol. 2, No. 1 Kagami: Control of the Marangoni Effect in the Drying Process...

\section{References}

Bornside, D. E., Macosko, C. W. and Scriven, L. E. (1989), "Spin coating: One-dimensional model", J. Appl. Phys., 66, 5185-5193.

de Gans, B. J. and Schubert, U. S. (2004), "Inkjet Printing of Well-Defined Polymer Dots and Arrays", Langmuir, 20, 7789-7793.

Hickman, K. C. D. and Trevoy, D. J. (1952), "Studies in High Vacuum Evaporation: The Falling-Stream Tensimeter", Ind. Eng. Chem., 44, 18821888.

Kagami, H. (2003), "A modified model of drying process of a polymer liquid film taking effects of latent heat and heat conductivity into account", Proc. SPIE., 5130, 220-227.

Kagami, H. (2004a), "The influence of spatio-temporal variation of temperature distribution in a polymer solution on a flat substrate on formation of polymer film's thickness distribution during drying process based on results of simulation of the modified model", Proc. SPIE., 5446, $135-142$.

Kagami, H. (2004b), "An additional modified model of drying process of a polymer liquid film - influence of an abrupt drop of vapor pressure peculiar to polymer solution -", The 9th Asia Pacific Physics Conference Proceedings, 10-10C.

Kagami, H. (2007), "Nonlinear dynamics that appears in the dynamical model of drying process of a polymer solution coated on a flat substrate", Proc. SPIE., 6417, 641704-1 - 8.

Kagami, H. (2008), "A modified dynamical model of drying process of polymer blend solution coated on a flat substrate", Proc. SPIE., 7028, 702825-1 - 9 .

Kagami, H. (2009), "Characteristic three-dimensional structure of resist's distribution after drying a resist solution coated on a flat substrate: analysis using the extended dynamical model of the drying process", Proc. SPIE., 7273, 727335-1 - 8 .

Kagami, H. (2010), "A modified dynamical model of drying process of a polymer solution having plural solvents coated on a flat substrate for a flat and homogeneous polymer film fabrication", Proc. SPIE., 7716, 771625-1 -9 .

Kagami, H. (2011), "Thickness control of a thin film after drying through thermal and evaporative management in drying process of a polymer solution coated on a flat substrate: application of the dynamical model of the drying process", Proc. SPIE., 8068, 806813-1 - 6.

Kagami, H. (2014a), "More minute thickness control of a thin film after drying through temperature, evaporation and concentration management in drying process of a polymer solution coated on a flat substrate", Proceedings of the 19th International Drying Symposium, Lyon, August 24-27, 2014, ISBN 978-2-7598-1631-6. 
Kagami, H. (2014b), "Impact of the Marangoni effect on the thin film thickness profile after drying polymer solution coated on a flat substrate", JPS Conf. Proc., 1, 015087-1 - 5.

Kagami, H. and Kubota, H. (2010a), "Applying the dynamical model of drying process of a polymer solution coated on a flat substrate to effects of bumpy substrate", Proc. SPIE., 7764, 77640T-1 - 8.

Kagami, H., Kubota, H. (2010b), "Thickness control of a thin film after drying through thermal management in drying process of a polymer solution coated on a flat substrate : application of the dynamical model of the drying process", Proceedings of the 2nd International Symposium on Thermal Design and Thermophysical Property for Electronics and Energy, 62-64.

Kagami, H., Kubota, H. (2011), "A dynamical model of drying process of a polymer solution having plural solvents and plural solutes (polymers) coated on a flat substrate for a flat and homogeneous polymer film fabrication", Physica Status Solidi, C 8, No. 2, 586-588.

Kagami, H., Miyagawa, R., Kawata, A., Nakashima, D., Kobayashi, S., Kitano, T., Takeshita, K., Kubota, H. and Ohmi, T. (2002), "A model of coating and drying process for the flat polymer film fabrication", Proc. SPIE., 4754, 252-259.

Routh, A. F. and Russel, W. B. (1998), "Horizontal drying fronts during solvent evaporation from latex films", AIChE J., 44, 2088-2098.

Soltman, D. and Subramanian, V. (2008), "Inkjet-Printed Line Morphologies and Temperature Control of the Coffee Ring Effect", Langmuir, 24, 22242231. 
